\title{
Modelling HIV dynamics with cell-to-cell transmission and CTL
} response

\author{
Zirui Zhu ${ }^{1}$, Ranchao $\mathrm{Wu}^{2}, \mathrm{Yu} \mathrm{Yang}^{3}$, and Yancong $\mathrm{Xu}^{1}$ \\ ${ }^{1}$ Hangzhou Normal University \\ ${ }^{2}$ Anhui University \\ ${ }^{3}$ Shanghai Lixin University of Accounting and Finance
}

September 24, 2021

\begin{abstract}
In most HIV models, the emergence of backward bifurcation means that the control for basic reproduction number less than one is no longer effective for HIV treatment. In this paper, we study an HIV model with CTL response and cell-to-cell transmission by using the dynamical approach. The local and global stability of equilibria is investigated, the relations of subcritical Hopf bifurcation and supercritical bifurcation points are revealed, especially, the so-called new type bifurcation is also found with two Hopf bifurcation curves meeting at the same Bogdanov-Takens bifurcation point. Forward and backward bifurcation, Hopf bifurcation, saddle-node bifurcation, Bogdanov-Takens bifurcation are investigated analytically and numerically. Two limit cycles are also found numerically, which indicates that the complex behavior of HIV dynamics. Interestingly, the role of cell-tocell interaction is fully uncovered, it may cause the oscillations to disappear and keep the so-called new type bifurcation persist. Finally, some conclusions and discussions are also given.
\end{abstract}

\section{Hosted file}

ZWX.pdf available at https://authorea.com/users/435854/articles/538593-modelling-hivdynamics-with-cell-to-cell-transmission-and-ctl-response

\section{Hosted file}

ZWXY.tex available at https://authorea.com/users/435854/articles/538593-modelling-hivdynamics-with-cell-to-cell-transmission-and-ctl-response 
figures/Fig1a/Fig1a-eps-converted-to.pdf 
figures/Fig1b/Fig1b-eps-converted-to.pdf 
figures/Fig2a/Fig2a-eps-converted-to.pdf 
figures/Fig2b/Fig2b-eps-converted-to.pdf 
figures/Fig3a/Fig3a-eps-converted-to.pdf 
figures/Fig3b/Fig3b-eps-converted-to.pdf 
figures/Fig3c/Fig3c-eps-converted-to.pdf 
figures/Fig3d/Fig3d-eps-converted-to.pdf 
figures/Fig4a/Fig4a-eps-converted-to.pdf 
figures/Fig4b/Fig4b-eps-converted-to.pdf 
figures/Fig5a/Fig5a-eps-converted-to.pdf 
figures/Fig5b/Fig5b-eps-converted-to.pdf 
figures/Fig6/Fig6-eps-converted-to.pdf 
figures/Fig7a/Fig7a-eps-converted-to.pdf 
figures/Fig7b/Fig7b-eps-converted-to.pdf 
figures/Fig7c/Fig7c-eps-converted-to.pdf 
figures/Fig8a/Fig8a-eps-converted-to.pdf 
figures/Fig8b/Fig8b-eps-converted-to.pdf 
figures/Fig9a/Fig9a-eps-converted-to.pdf 
figures/Fig9b/Fig9b-eps-converted-to.pdf 
figures/Fig10/Fig10-eps-converted-to.pdf 